\title{
Denominators count: supplementing surveillance data for genital Chlamydia trachomatis infection with testing data, Norway, 2007 to 2013
}

H Kløvstad ${ }^{1}$, P Aavitsland ${ }^{2}$

1. Norwegian Institute of Public Health, Department of Infectious Disease Epidemiology, Oslo, Norway

2. Epidemi, Lasarettet, Kristiansand, Norway

Correspondence: Hilde Kløvstad (hilde.klovstad@fhi.no)

Citation style for this article:
Kløvstad H, Aavitsland P. Denominators count: supplementing surveillance data for genital Chlamydia trachomatis infection with testing data, Norway, 2007 to 2013. Euro Surveill. 2015;20(36):pii=30012. DOI: http://dx.doi.org/10.2807/1560-7917.ES.2015.20.36.30012

Article submitted on 22 October 2014 / accepted on 03 July 2015 / published on 10 September 2015

As genital Chlamydia trachomatis (chlamydia) infection is often asymptomatic, surveillance of diagnosed cases is heavily influenced by the rate and distribution of testing. In 2007, we started supplementing case-based surveillance data from the Norwegian Surveillance System for Communicable Diseases (MSIS) with aggregated data on age group and sex of individuals tested. In this report, annual testing rates, diagnosis rates and proportion positive for chlamydia in Norway between 1990 and 2013 are presented. From 2007, rates are also stratified by age group and sex. The annual testing rate for chlamydia culminated in the early 1990 s, with 8,035 tested per 100,000 population in 1991. It then declined to 5,312 per 100,000 in 2000 after which it remained relatively stable. Between 1990 and 2013 the annual rate of diagnosed cases increased 1.5 times from ca 300 to ca 450 per 100,000 population. The proportion of positive among the tested rose twofold from ca $4 \%$ in the 1990 s to $8 \%$ in 2013. Data from 2007 to 2013 indicate that more women than men were tested (ratio: 2.56 ; $95 \%$ confidence interval (CI): 2.56-2.58) and diagnosed (1.54; $95 \% \mathrm{Cl}: 1.52-1.56)$. Among tested individuals above 14 years-old, the proportion positive was higher in men than women for all age groups. Too many tests are performed in women aged 30 years and older, where 49 of 50 tests are negative. Testing coverage is low (15\%) among 15 to 24 year-old males. Information on sex and age-distribution among the tested helps to interpret surveillance data and provides indications on how to improve targeting of testing for chlamydia. Regular prevalence surveys may address remaining limitations of surveillance.

\section{Introduction}

Genital Chlamydia trachomatis infection (hereafter: chlamydia) is the most frequently reported sexually transmitted infection (STI) in Europe, in particular in Norway $[1,2]$. Early diagnosis and treatment has been considered a major strategy to prevent complications and further transmission of chlamydia [3,4], and countries have tried various strategies to increase testing in target populations [5]. The evidence for the effect of early diagnosis and treatment of chlamydia on the population level is however weak $[6,7]$.

In Norway, there is no opportunistic or systematic screening programme for chlamydia. However, sampling for chlamydia testing is available at no extra cost in all general practitioners' offices, hospitals, STI clinics, students' clinics, youth clinics (ca 350), and other sites. Individuals can also order sampling kits on the Internet and send samples to a diagnostic laboratory by mail. Since 1995 the following groups are recommended to be tested: any person below 25 years of age after each change of sexual partner, individuals with clinical symptoms compatible with chlamydia or epidemiological link to another case, women below 25 years of age during antenatal care and women undergoing legal abortion [8-12]. Recommendations for annual testing of chlamydia and other STIs in men who have sex with men were issued in 2005 [11].

Since 2005 , laboratories in Norway are mandated to report limited data (age and sex) on cases of chlamydia infection to the Norwegian Surveillance System for Communicable Disease (MSIS) which is owned by the Ministry of Health and operated by the Norwegian Institute of Public Health (NIPH) [2]. Interpretation of chlamydia surveillance data is challenging as many asymptomatic cases remain undiagnosed and unreported. The reported number of diagnosed cases is thus a result of both the incidence of chlamydia and the testing policies and practices. For this reason, we augmented the laboratory case-based surveillance system [2] with a voluntary collection of the laboratories' data on sex and age group distribution of all individuals tested for chlamydia. 


\section{FIGURE 1}

Annual genital Chlamydia trachomatis testing rates (per 10,000 population) and diagnosis rates (per 100,000 population), Norway, 1990-2013

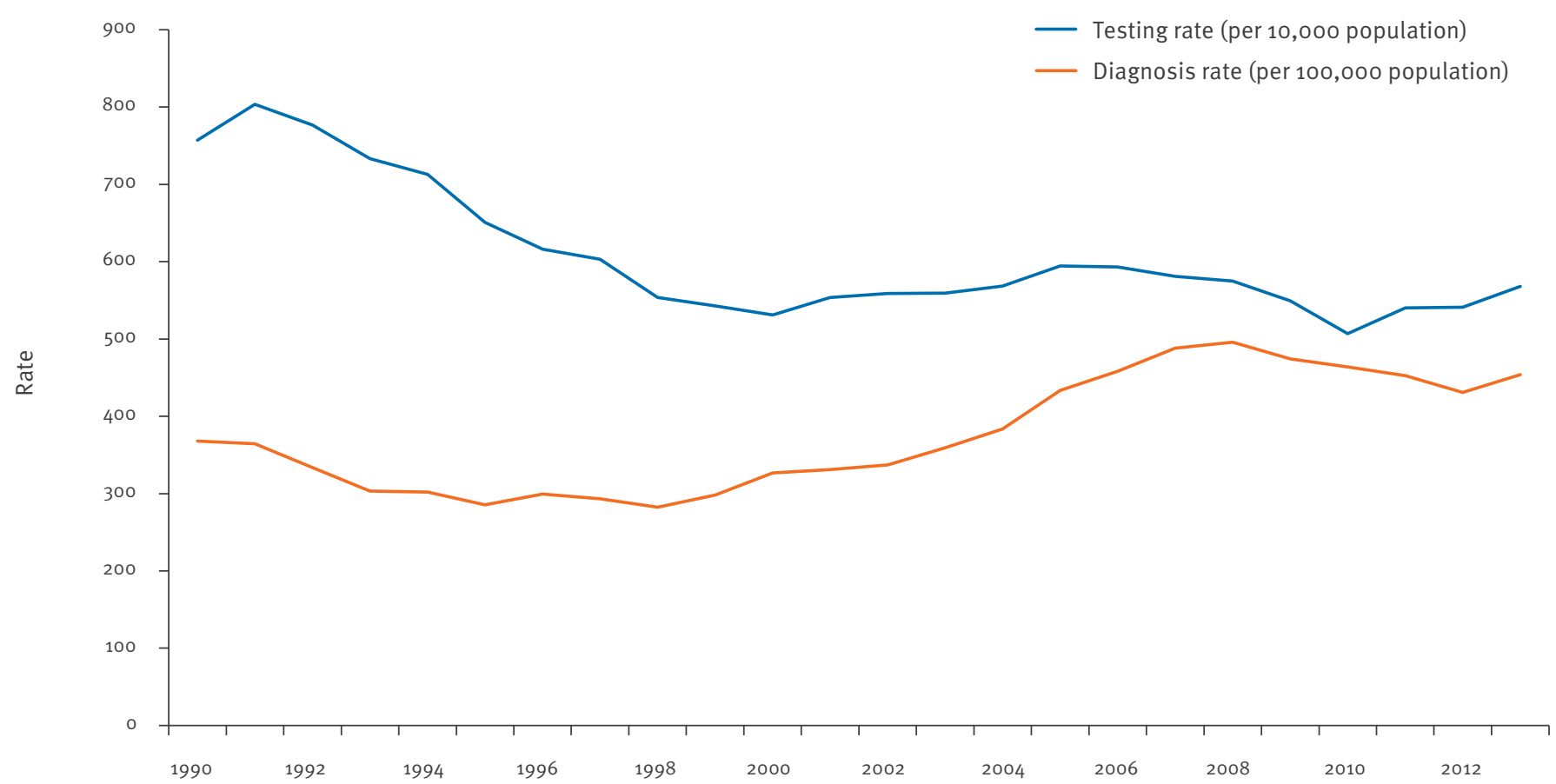

The objective of this study was to describe testing patterns for genital chlamydia infection in Norway and incidence of diagnosed genital chlamydia infection in order to better understand the chlamydia epidemic and improve targeting of chlamydia testing in the future.

\section{Methods}

\section{Mandatory surveillance}

Before 2005, laboratories reported the aggregated number of chlamydia tests performed and the number of positive test results on a yearly basis. Since 2005 , laboratories have been mandated to report case-based data in February for the preceding year. The following variables are collected for each diagnosed case; date of diagnosis, birth year, sex and municipality of residence [2]. Each case represents one record in an Excel file, which is encrypted and password protected and sent by mail to NIPH. No unique identifiers are used. Clinicians do not report.

A case is defined as a person with one or more positive laboratory tests for Chlamydia trachomatis in a urinary sample or a sample from anus, cervix, urethra, or vagina within a period of 60 days. This case definition is used to avoid counting as a new case tests taken from multiple sites or a positive test of cure (which up until 2013 was universally recommended to be taken 5-6 weeks after treatment [2], now only if poor compliance is suspected, if symptoms persist, if reinfection is probable, or if patient is pregnant $[10,11])$.
The total number of chlamydia tests performed in a year by each laboratory is also collected, using the same principles defining a case; 'one tested' refers to one person with one or more chlamydia tests within a period of 60 days. The total number tested (one number=denominator) during the year is reported in the cover letter attached to the Excel file.

\section{Voluntary supplementary data collection}

In 2007, we augmented surveillance with a voluntary annual aggregate reporting from laboratories of sex and age group distribution of all tested. The table is sent by email to NIPH. Participation has increased year by year. Information on sex and age distribution among the tested was received from 10/22 laboratories in 2007, 7/22 laboratories in 2008, 13/20 laboratories in 2009, 16/19 laboratories in 2010, 17/19 laboratories in 2011, 17/19 laboratories in 2012, and in 2013 16/18 laboratories. For the analyses, we have assumed that the age and sex distribution in the non-reporting laboratories was the same as in the reporting laboratories and extrapolated these distributions to the reported sample of all tested. To justify this assumption, we compared the proportion of 20 to 24 year-old women among all those tested in one laboratory in its first reporting year to the corresponding proportion in all the other laboratories reporting that year. We found that the difference was usually in the order of zero to three per cent points. 


\section{FIGURE 2}

Annual genital Chlamydia trachomatis diagnosis rates (per 100,000 population) by age group and sex, Norway, 2007-2013

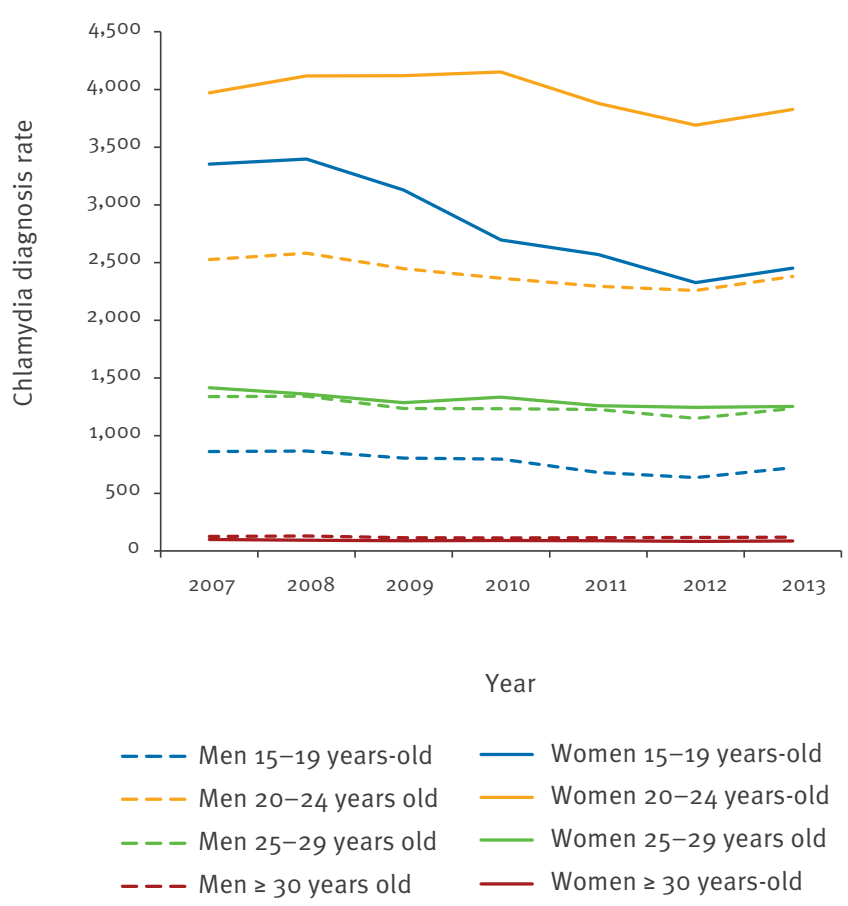

\section{FIGURE 3}

Annual genital Chlamydia trachomatis testing rates (per 100 population) by age group and sex, Norway, 2007-2013

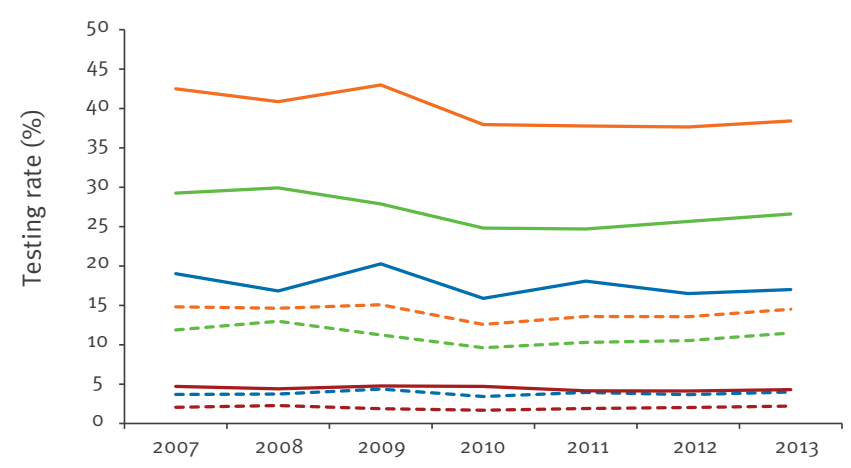

Year

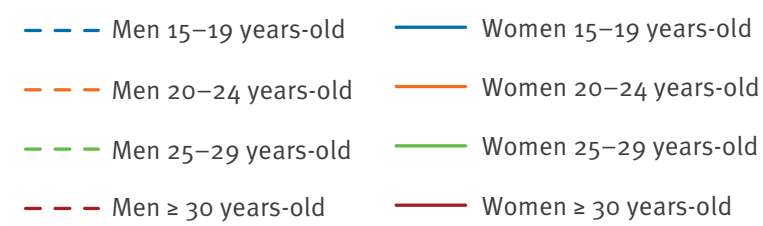

\section{Data analyses}

We present annual testing rates, diagnosis rates and proportion positive for the total population (19902013) and by age group and sex (2007-2013). Yearly population data are collected from Statistics Norway. For testing of trends, we used a negative binomial regression with a year covariate.

\section{Results}

\section{Rates of chlamydia diagnosis}

Between 1990 and 2013 the annual number of diagnosed chlamydia cases in Norway per population was multiplied 1.5 times from ca 300 to ca 450 per 100,000 per year. The sharpest rise in this period corresponded to a 1.7 times increase from 293 per 100,000 population in 1997 to 496 in 2008. In 2013 the diagnosis rate was 454 per 100,000 population (Figure 1). A trend test of the diagnosis rate showed an average increase of $2.2 \%(95 \% \mathrm{Cl}: 1.4-2.9 \%)$ each year from 1990 to 2013 (p-value<0.001).

For the 2007 to 2013 period, when data on age group and sex were available among the tested, the highest diagnosis rates were found in 20 to 24 year-old women, followed by women aged 15 to 19 years and men aged 20 to 24 years. In 2010, the diagnosis rate among women aged 20 to 24 was 4,151 per 100,000, which is equivalent to one in 25 women having been diagnosed with chlamydia that year. Apartfor some declines until 2012 among women aged 15 to 19 years-old (from 2008) and 20 to 24 years-old (from 2010), the diagnosis rates have been relatively stable in the population for the whole period considered (2007-2013). (Figure 2).

\section{Rates of chlamydia testing}

The testing rate for chlamydia in Norway was at its highest in the beginning of the 1990 s with a peak of 8,035 tested per 100,000 population in 1991 . The testing rate then decreased yearly and reached 5,312 tested per 100,000 in year 2000 . Since 2000 , the testing rate for chlamydia in Norway has been relatively stable (Figure 1).

According to the data from 2007 onwards, among individuals over 14 years-old, women have higher testing rates than men in all respective age groups (Figure 3 ). These and age group specific testing rates have been relatively stable throughout the 2007 to 2013 period.

The chlamydia testing coverage (defined as the number of tests divided by the number of individuals in the population considered) was $38 \%(63,679 / 165,558)$ in women aged 20 to 24 years in 2013 while the corresponding figure for men in the same age group was $15 \%(25,167 / 173,489)$. In the age group 15 to 19 years the coverage was $4 \%(6,687 / 168,038)$ and $17 \%$ $(26,834 / 158,031)$ for men and women, respectively.

\section{Proportion positive for chlamydia among the tested}

In 1990 the proportion positive among those tested for chlamydia was $4.9 \%(15,567 / 320,459)$. The proportion positive among those tested for chlamydia decreased to $4.1 \%(13,033 / 315,257)$ in 1993 (when the testing rate was 733 per 100,000 population), and increased yearly to a peak of $9.1 \%(22,527 / 246,268)$ in 2010 (Figure 4) before going down to $8.0 \%(22,946 / 286,653)$ in 2013 
Annual rates of genital Chlamydia trachomatis testing (per 100,000 population) and annual proportion of positive test results, Norway, 1988-2013

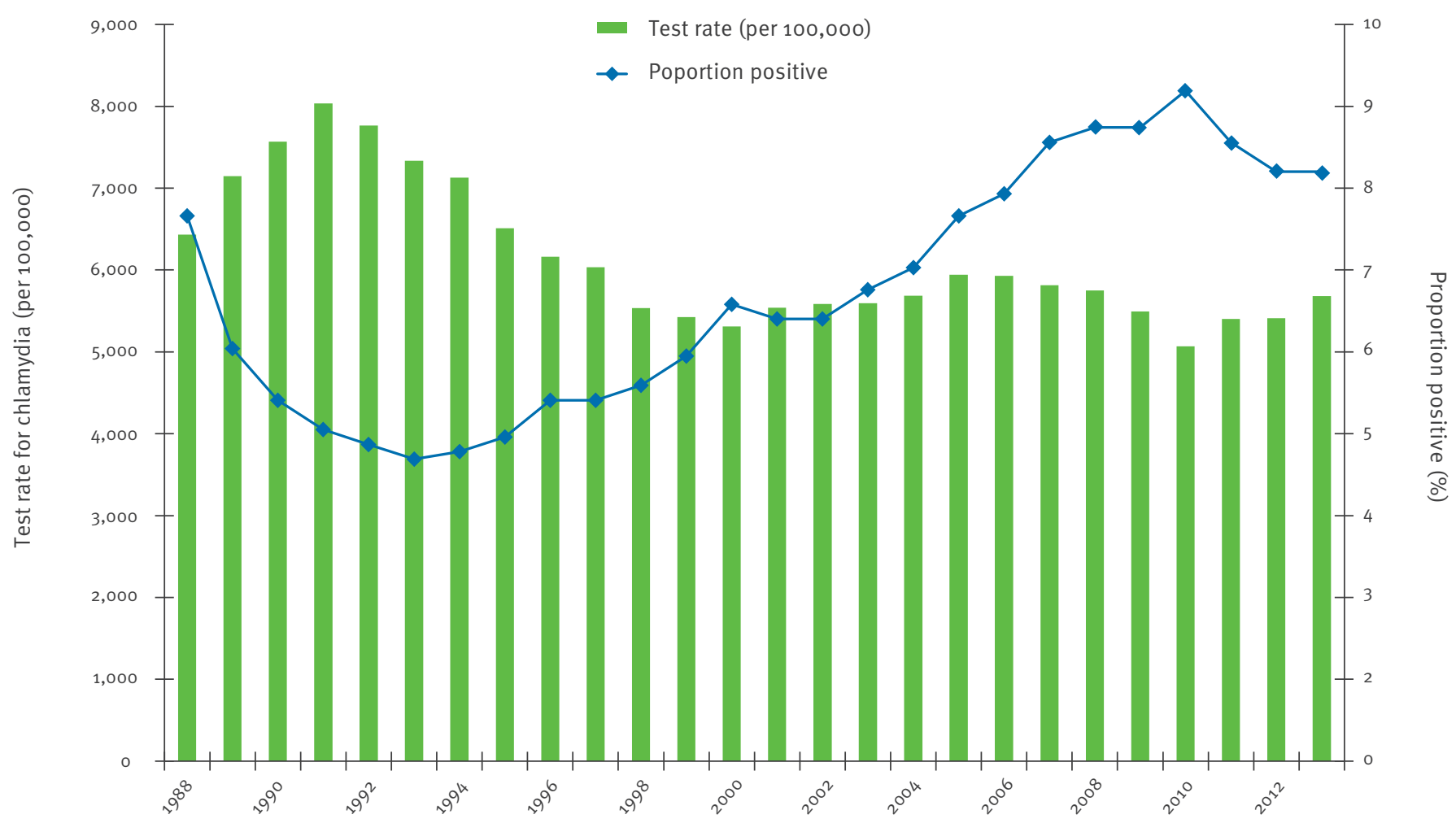

The peak in 2010 coincided with a drop by $6.6 \%$ in the number of people tested.

Throughout the 2007 to 2013 period, the proportions of positive test results for chlamydia among men or women tested were respectively highest in the youngest age groups (15-19 years), especially among men (Figure 5). In the whole period, there was large variation in proportion positive, e.g. $17.7 \%$ among men aged 15 to 19 years to $2.1 \%$ among women 30 years of age or older (Table).

\section{Sex and age distribution among the tested and} diagnosed

In the 2007 to 2013 period, women made up $72 \%$ $(1,031,667 / 1,433,258)$ of those tested for chlamydia and $61 \%(71,538 / 117,978)$ of those diagnosed (Table). Women were 2.56 times $(95 \% \mathrm{Cl}: 2.56-2.58)$ more likely to be tested than men and 1.54 times $(95 \% \mathrm{Cl}$ : 1.52-1.56) more likely to be diagnosed. In the age group 15 to 19 years, women were 4.56 (95\% Cl: $4.53-$ 4.61) times more likely to be tested than men.

The majority of chlamydia tests are taken in persons 25 years of age and older while the majority of diagnoses are found in persons younger than 25 years. Among women, $45 \%(466,655 / 1,031,667)$ of the tested were less than 25 years-old while $75 \%(53,407 / 71,538)$ of the diagnosed were below this age. Men under 25 years-old represented $38 \%(153,033 / 401,591)$ of all tested men and $56 \%(26,090 / 46,440)$ of all diagnosed men (Table). For women aged 30 years and older, only $2.1 \%(7,270 / 349,385)$ of those tested were positive, which corresponds to 49 of 50 tests in this age group being negative. The sex and age distribution among the tested has remained stable throughout this seven year period.

\section{Discussion}

We have found that information on age group and sex among those who are tested for chlamydia helps us interpret the results obtained by the national surveillance system for chlamydia. The proportion positive among the tested rose twofold from around $4 \%$ in the 1990 to $8 \%$ in 2013 whereas the number of diagnosed cases per population was multiplied 1.5 times from ca 300 to ca 450 per 100,000 per year in the same period. One possible explanation is that the true incidence and prevalence of chlamydia has indeed increased. Recommendations for chlamydia testing were issued in 1995 [12]. Another possible explanation could therefore be that chlamydia testing, which has been reduced during the study period (ca 7,500 annual tests per 100,000 in 1990 to 5,500 in 2013), has become more targeted at 


\section{FIGURE 5}

Proportion of positive for genital Chlamydia trachomatis among persons tested, by age group and sex, Norway, 2007-2013

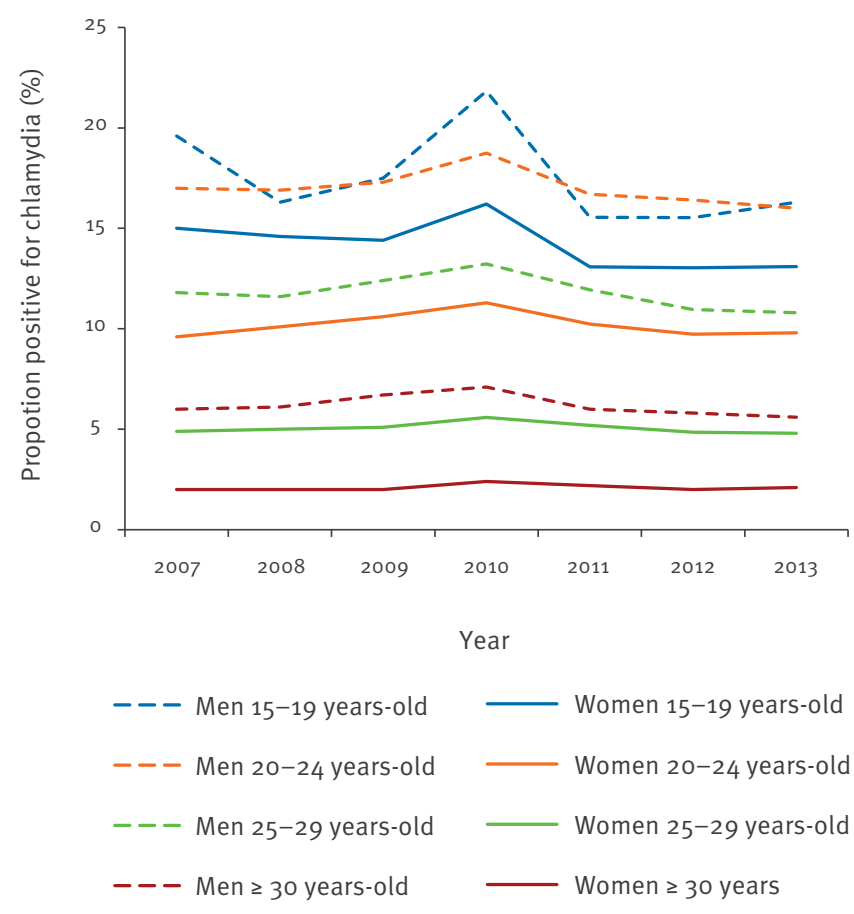

high-prevalence groups so that the proportion positive among the tested has increased. Finally, the introduction of more sensitive diagnostic methods in the late 1990 s may have contributed to the increase in positivity. An association was found between the introduction of nucleic acid amplification test (NAAT) and the proportion positive for chlamydia among the tested in Norway [2]. However, as the positivity rate continued to rise even after the introduction of NAAT, other factors may be have been more important to explain the observed increase, such as secular trend and case mix among the tested.

\section{The value of testing data}

The surveillance data reflects the sex distribution among those who get tested for chlamydia in Norway. Among persons below 25 years of age, more women get tested, thus more women get diagnosed with chlamydia than men. Women aged 20 to 24 years constitute the group with the highest testing and diagnosis rates for chlamydia in Norway. The testing rates among the males in this age group are lower, hence, the incidence rates are lower. More women than men get tested also among persons above 25 years of age, but the sex distribution among the diagnosed is more equal. Among those over 14 years-old, the proportion positive is higher among men than women in all age groups, which probably reflects that men who are offered or choose to get tested for chlamydia are more at risk of infection (partner tracing, symptoms, men who have sex with men), not that the chlamydia prevalence is higher in men than in women [13].
Proportion positive among the tested is an important supplement that helps us understand the observed results of diagnosed chlamydia cases. In the early 2000 , the positivity rate increased in parallel to the rise in diagnosis rate. These increases could not be explained by more testing in this period as this was rather stable. Since 2007 , we have observed a decline in diagnosed cases among the 15 to 19 year-olds, especially among girls. Testing data show that this decline only partly can be attributed to less testing in this age group. The proportion positive has also decreased in the same period. This could indicate that the chlamydia prevalence in this age group has dropped. It could, however, also indicate that that we do not reach those most at risk in this age group. There are no available data to support a change is sexual behaviour or in chlamydia prevalence for the youngest age group in this period. A decline in diagnosed cases among those below 25 years of age has also been observed in our neighbouring country Denmark since 2009 [14].

\section{Comparison with other countries}

The testing rate (and diagnosis rate) in Norway is similar to the testing rates in Sweden and Denmark. In 2012 , the testing rates per 100,000 total population was 4,862 and 6,087 in Sweden and Denmark respectively $(5,461$ in Norway) $[14,15]$. The sex distribution among the tested is also comparable [16]. In several other European countries, the reported testing rates are much lower [5]. Comparing testing rates in different European countries is however challenging due to the variation of systems collecting data on the number of tested.

\section{How data on chlamydia testing help us further target testing}

Testing data show that Norwegian women between 20 and 24 years-old adhere to the recommendations for testing. In this group, the testing rate corresponds to coverage of close to $40 \%$ if there were no repeat testing, compared with $14 \%$ for men in the same age group. The chlamydia testing coverage in England in 2012 for the 15 to 24 year-old age group was $35 \%$ for women and $15 \%$ for men [17]. Corresponding figures from Norway were $28 \%$ and $9 \%$ for women and men, respectively. A register study from Central Norway (1990-2003) showed that $85 \%$ of women had had at least one chlamydia test before the age of 25 years [18]. More than half of these were registered with two or more tests within an observation period of maximum four years. The results confirm a high coverage of chlamydia testing among women in their early twenties [18].

One possible explanation for the sex difference could be that women take more concern for their sexual health, for instance seeking guidance for birth control, and therefore have a more active health seeking behaviour than men. Supporting this assumption are two Norwegian studies showing that women diagnosed with chlamydia are more likely to get their prescribed treatment than men diagnosed with chlamydia $[19,20]$. 
Number and proportion tested and diagnosed for genital Chlamydia trachomatis by age group and sex, Norway, 2007-2013

\begin{tabular}{|l|c|c|c|c|c|c|c|c|c|c|}
\hline \multirow{2}{*}{$\begin{array}{l}\text { Age groups } \\
\text { in years }\end{array}$} & $\begin{array}{c}\text { Number } \\
\text { tested }\end{array}$ & $\begin{array}{c}\text { Proportion } \\
\text { of all } \\
\text { women } \\
\text { tested (\%) }\end{array}$ & $\begin{array}{c}\text { Number } \\
\text { diagnosed }\end{array}$ & $\begin{array}{c}\text { Proportion } \\
\text { of all } \\
\text { women } \\
\text { diagnosed } \\
(\%)\end{array}$ & $\begin{array}{c}\text { Proportion } \\
\text { positive } \\
(\%)\end{array}$ & $\begin{array}{c}\text { Number } \\
\text { tested }\end{array}$ & $\begin{array}{c}\text { Proportion } \\
\text { of all men } \\
\text { tested (\%) }\end{array}$ & $\begin{array}{c}\text { Number } \\
\text { diagnosed }\end{array}$ & $\begin{array}{c}\text { Proportion } \\
\text { of all men } \\
\text { diagnosed } \\
(\%)\end{array}$ & $\begin{array}{c}\text { Proportion } \\
\text { positive (\%) }\end{array}$ \\
\hline $0-14$ & 2,743 & 0.3 & 94 & 0.1 & 3.4 & 810 & 0.0 & 16 & 0.0 & 2.0 \\
\hline $15-19$ & 144,525 & 14.0 & 20,645 & 28.8 & 14.3 & 33,616 & 8.4 & 5,935 & 12.8 & 17.7 \\
\hline $20-24$ & 319,380 & 31.0 & 32,668 & 45.6 & 10.2 & 118,607 & 29.5 & 20,139 & 43.3 & 17.0 \\
\hline $25-29$ & 215,625 & 20.9 & 10,861 & 15.2 & 5.0 & 91,983 & 22.9 & 10,776 & 23.2 & 11.7 \\
\hline$\geq 30$ & 349,385 & 33.9 & 7,270 & 10.2 & 2.1 & 156,575 & 39.0 & 9,574 & 20.6 & 6.1 \\
\hline Total & $\mathbf{1 , 0 3 1 , 6 6 7}$ & 100 & $\mathbf{7 1 , 5 3 8}$ & 100 & 6.9 & $\mathbf{4 0 1 , 5 9 1}$ & 100 & 46,440 & 100 & 11.6 \\
\hline$<25$ & 466,656 & 45.2 & 53,407 & 74.6 & 11.4 & 153,033 & 38.1 & 26,090 & 56.1 & 17.0 \\
\hline$\geq 25$ & 565,011 & 54.8 & 18,131 & 25.3 & 3.2 & 248,559 & 61.9 & 20,350 & 43.8 & 8.2 \\
\hline Total & $\mathbf{1 , 0 3 1 , 6 6 7}$ & 100 & $\mathbf{7 1 , 5 3 8}$ & 100 & 6.9 & $\mathbf{4 0 1 , 5 9 1}$ & 100 & 46,440 & 100 & 11.6 \\
\hline
\end{tabular}

In the youngest age group (15-19 years) the testing rate is lower. The results of different sexual behaviour studies conducted in the period between 1987 and 2006 in Norway inform of a mean age of sexual debut varying from 16 to 18 years, lower for women than men and decreasing over time $[13,21,22]$. Many young people in this age group are not yet sexually active and at risk of chlamydia. This may contribute to a lower testing rate in this age group. The high positivity rate among the tested in this age group may indicate that testing occurs among those at high risk.

The majority of tests are performed in persons 25 years and above while the majority of the diagnosed cases are found in the younger age groups. Among women above 30 years-old the proportion positive is very low. Widespread testing in such a low prevalence population means waste of resources and low predictive value of a positive test result. Situations where unnecessary chlamydia testing outside the recommended indications could take place are when women above 30 have a smear test taken as part of the cancer screening programme (every third year [23]), during consultations for contraception and during antenatal care.

The high proportion positive among men between 25 and 40 years-old ( $12 \%$ in men aged $25-29$ years) indicates that chlamydia testing is rather well targeted and not only routine testing in this group. Patients diagnosed with chlamydia have the responsibility to cooperate in partner tracing, according to the Norwegian Infectious Disease Control Act [24]. Although indications for testing are not known, partner tracing is likely to be a common indication for testing. A study from Sweden showed that partner tracing was the most common reason for testing among men [16]. Due to the lack of prevalence surveys, we do not know if the chlamydia prevalence is higher among men than women in this age group. However, studies from other countries have shown that the peak prevalence age among men is higher than among women [25]. Along with the high proportion positive found among men of 25 to 29 years of age, this could be an incentive to explore whether recommendations for chlamydia testing also should include men up to 30 years.

Use of selective screening criteria to increase the diagnostic yield among the tested, even within the groups recommended for chlamydia testing, has not been applied in Norway. Such approach may be valuable $[26,27]$ although selective screening criteria have shown difficult to identify $[28,29]$.

\section{Limitations}

Data on chlamydia testing has improved our understanding of the chlamydia surveillance data and of how targeted chlamydia testing is. However, these data merely reflect who gets an offer or decides to get tested and do not necessarily bring us closer to the real chlamydia distribution in the population. We do not know which healthcare providers have ordered the tests or their indications. This could have provided useful information to explain the sex differences in testing rates.

The case definitions used for both a diagnosed case and a tested person include only one count within a period of 60 days. In the absence of a unique identifier, this is done to avoid counting twice those who take samples from more than one site or who return for a test of cure. There is a problem with both the sensitivity and specificity of this case definition as 60 days is a longer time period than the recommendations for test of cure. If a person is re-infected within a period of 60 days, it will not be counted as a double incident of chlamydia, only one. The extent of possible double reporting or underreporting of double chlamydia incidents is not known. 
Due to the absence of a unique identifier and the aggregation of test data, we are also not able to detect re-testing or re-infections with our surveillance system. As testing for chlamydia is recommended in the under 25 year-olds between each partner change, probably, some individuals are tested more than once per year. Therefore, converting an annual age group specific testing rate to a testing coverage in that age group probably leads to overestimated coverage percentages. Currently, there are no recommendations for retesting in Norway.

We have extrapolated the sex and age distribution from the reported sample to all tested. This may introduce bias if the sex and age distribution of those who get tested correlates with characteristics in the unreported sample - for example with geographical area. Fortunately, the completeness of data on testing has increased to over $90 \%$ in the last few years, reducing the possibility for bias.

\section{Conclusion}

Augmenting case-based chlamydia surveillance with aggregated data on age group and sex of all who have been tested helps in the interpretation of surveillance data. We have found that more women than men are tested for chlamydia in Norway. This partly explains the higher diagnosis rates among women. Too many tests are performed in groups with very low prevalence of infection, giving a very low yield. Still, surveillance data reflect those who get tested, and not necessarily the real distribution of cases. Regular population based prevalence surveys would be a useful supplement.

\section{Acknowledgements}

We gratefully acknowledge all laboratories reporting chlamydia data in Norway. The research was funded by the Norwegian Institute of Public Health.

\section{Conflict of interest}

None declared.

\section{Authors' contributions}

HK has been involved in planning and setting up the presented surveillance scheme, has done the quality checks of surveillance data, the analysis of and interpretation of data and drafted and revised the manuscript. PAa has been involved in the planning and design of the surveillance scheme, has participated in the analysis and interpretation of data and has made a substantial contribution to the drafting and revision of the manuscript. All authors have read and approved the final manuscript.

\section{References}

1. European Centre for Disease Prevention and Control (ECDC). Annual epidemiolgical report. Reporting on 2011 surveillance data and 2012 epidemic intelligence data. Stockholm: ECDC; 2013.

2. KløvstadH, AavitslandP. Chlamydia trachomatis infections in norway, 1986 to 2006, surveillance data.Sex Transm Dis.
2009;36(1):17-21. DOI: 10.1097/OLQ.ob013e31818653bo PMID: 18923334

3. U.S. Preventive Services Task Force,. Screening for chlamydial infection: U.S. Preventive Services Task Force recommendation statement.Ann Intern Med. 2007;147(2):128-34. DOI: 10.7326/0003-4819-147-2-200707170-00172 PMID: 17576996

4. Centers for Disease Control and Prevention (CDC),. CDC Grand Rounds: Chlamydia prevention: challenges and strategies for reducing disease burden and sequelae.MMWR Morb Mortal Wkly Rep. 2011;60(12):370-3.PMID: 21451447

5. European Centre for Disease Prevention and Control (ECDC). Chlamydia control in Europe - a survey of Member States. Stockholm: ECDC; 2014.

6. LowN, BenderN, NarteyL, ShangA, StephensonJM. Effectiveness of chlamydia screening: systematic review.Int J Epidemiol. 2009;38(2):435-48. DOI: 10.1093/ije/dyn222 PMID: 19060033

7. European Centre for Disease Prevention and Control (ECDC). Chlamydia control in Europe: literature review. Stockholm: ECDC; 2014.

8. Norsk gynekologisk forening. Veileder i fødselshjelp. [Guide for obstetrics]; 2008. Norwegian. Available from: http://www.helsebiblioteket.no/retningslinjer/gynekologi/ f\%C3\%B8dselshjelp-veileder-i-f\%C3\%B8dselshjelp-2008

9. The Norwegian Directorate for Health and Social Affairs. Nasjonale faglige retningslinjer for svangerskapsomsorgen. [National Clinical Guideline for Antenatal Care]; 2006. Available from: https://helsedirektoratet.no/Lists/Publikasjoner/ Attachments/393/nasjonal-faglig-retningslinje-forsvangerskapsomsorgen-fullversjon.pdf

10. The Norwegian Directorate of Health. Nasjonale faglige retningslinjer for antibiotikabruk i primærhelsetjenesten. [National professional guidelines for the use of antibiotics in primary care]; 2013. Norwegian. Available from: http://www. helsebiblioteket.no/retningslinjer/antibiotika/forord

11. The Norwegian Institute of Public Health. Smittevernboka. [National Infection control Guidance]. Available from: http:// www.fhi.no/publikasjoner-og-haandboker/smittevernboka

12. AavitslandP, LystadA. [Indications for testing for sexually transmitted Chlamydia trachomatis infections]. Tidsskr Nor Laegeforen. 1995;115(25):3141-4.PMID: 8539697

13. KløvstadH, GrjibovskiA, AavitslandP. Population based study of genital Chlamydia trachomatis prevalence and associated factors in Norway: a cross sectional study.BMC Infect Dis. 2012;12(1):150. DOI: 10.1186/1471-2334-12-150 PMID: 22747602

14. Statens Serum Institut. Chlamydia 2012. EPI-NEWS. 2013; 36.

15. The Swedish Institute for Communicable Disease Control (SMI). Epidemiologisk årsrapport 2012. [Epidemiological Annual Report 2012]. Stockholm: SMI; 2013. Swedish.

16. LöfdahIM, RydevikG, BlaxhultA, HerrmannB. [Chlamydia infection among Swedish women. Contact tracing and reporting routines must be improved]. Lakartidningen. 2008;105(44):3116-20.PMID: 19031978

17. Wise J. Only $15 \%$ of young men in England were tested for chlamydia last year despite recommendations. BMJ. 2014;348(jun18 25):g4121.

18. BakkenIJ, NordbøSA, SkjeldestadFE. Chlamydia trachomatis testing patterns and prevalence of genital chlamydial infection among young men and women in central Norway 19902003: a population-based registry study.Sex Transm Dis. 2006;33(1):26-30. DOI: 10.1097/01.olq.0000187929.36118.d2 PMID: 16385219

19. KløvstadH, NatåsO, TverdalA, AavitslandP. Systematic screening with information and home sampling for genital Chlamydia trachomatis infections in young men and women in Norway: a randomized controlled trial.BMC Infect Dis. 2013;13(1):30. DOI: 10.1186/1471-2334-13-30 PMID: 23343391

20. GhaderiS, NordbøSA, BakkenIJ. [Chlamydia infections in South Trøndelag--treatment and follow-up]. Tidsskr Nor Laegeforen. 2011;131(5):461-3. DOI: 10.4045/tidsskr.10.0384 PMID: 21383798

21. Træen B, Stigum H, Magnus P. Rapport fra seksualvaneundersøkelsene 1987, 1992, 1997 og 2002. [Report of sexual behaviour surveys in 1987, 1992, 1997 and 2002]. Oslo: Norwegian Institute of Public Health; 2003. Norwegian.

22. PedersenW, SamuelsenSo. [New patterns of sexual behaviour among adolescents]. Tidsskr Nor Laegeforen. 2003;123(21):3006-9.PMID: 14618164

23. Kreftregisteret. Masseundersøkelser mot livmorhalskreft 2013. [Mass screening for cervical cancer]. Norwegian. Available from: http://kreftregisteret.no/no/Forebyggende/Nyhetssaker/

24. Lov om vern mot smittsomme sykdommer. [Communicable disease control act]. LOV-1994-08-05-55. Norwegian. 
25. SchmidBV, OverEA, van den BroekIV, Op de CoulEL, van BergenJE, FennemaJS, et al. Effects of population based screening for Chlamydia infections in the Netherlands limited by declining participation rates. PLoS ONE. 2013;8(3):e58674. DOI: 10.1371/journal.pone.0058674 PMID: 23527005

26. van den BroekIV, BrouwersEE, GötzHM, van BergenJE, Op de CoulEL, FennemaJS, et al. Systematic selection of screening participants by risk score in a Chlamydia screening programme is feasible and effective. Sex Transm Infect. 2012;88(3):205-11. DOI: 10.1136/sextrans-2011-050219 PMID: 22215696

27. GötzHM, van KlaverenD. Use of prediction rules in control of sexually transmitted infections: challenges and chances.Sex Transm Dis. 2014;41(5):331-2. DOI: 10.1097/ OLQ.0000000000000128 PMID: 24722389

28. Falasinnu T, Gilbert M, Salway TH, Gustafson P, Ogilvie G, Shoveller J. Predictors identifying those at increased risk for STDs: a theory-guided review of empirical literature and clinical guidelines. Int J STD AIDS. 2014

29. van ValkengoedIG, MorréSA, van den BruleAJ, MeijerCJ, DevilléW, BouterLM, et al. Low diagnostic accuracy of selective screening criteria for asymptomatic Chlamydia trachomatis infections in the general population. Sex Transm Infect. 2000;76(5):375-80. DOI: 10.1136/sti.76.5.375 PMID: 11141855 